Journal for ImmunoTherapy of Cancer

\section{Conditional immune toxicity rate in patients with metastatic renal and urothelial cancer treated with immune checkpoint inhibitors}

To cite: Nuzzo PV, Pond GR, Abou Alaiwi S, et al. Conditional immune toxicity rate in patients with metastatic renal and urothelial cancer treated with immune checkpoint inhibitors. Journal for ImmunoTherapy of Cancer 2020;8:e000371. doi:10.1136/jitc-2019-000371

- Additional material is published online only. To view please visit the journal online (http://dx.doi.org/10.1136/jitc2019-000371).

PVN and GRP contributed equally.

Society for Immunotherapy of Cancer (SITC) annual meetting 2019 in National Harbor, Maryland, USA and Merit Award at ASCO Genitourinary Cancers Symposium 2020, San Francisco, CA, USA.

Accepted 03 March 2020

Check for updates

(c) Author(s) (or their employer(s)) 2020. Re-use permitted under CC BY-NC. No commercial re-use. See rights and permissions. Published by BMJ.

For numbered affiliations see end of article.

\section{Correspondence to}

Dr Guru Sonpavde;

gurup_sonpavde@dfci.harvard. edu

\section{ABSTRACT}

Background Immune checkpoint inhibitors (ICls) are associated with immune-related adverse events (irAEs). Although the incidence and prevalence of irAEs have been well characterized in the literature, less is known about the cumulative incidence rate of irAEs. We studied the cumulative incidence of irAEs, defined as the probability of irAE occurrence over time and the risk factors for irAE development in metastatic urothelial carcinoma (mUC) and renal cell carcinoma (mRCC) patients treated with ICls. Methods We identified a cohort of patients who received ICls for mUC and mRCC. irAEs were classified using Common Terminology Criteria for Adverse Event (CTCAE) V.5.0 guidelines. The monthly incidence of irAEs over time was reported after landmark duration of therapy. Cumulative incidence of irAEs was calculated to evaluate the time to the first occurrence of an irAE accounting for the competing risk of death. Prognostic factors for irAE were assessed using the Fine and Gray method. Results A total of 470 patients were treated with ICls between July 2013 and October 2018 (mUC: 199 (42.3\%); mRCC: $271(57.7 \%)) .341$ (72.6\%) patients received monotherapy, 86 (18.3\%) received ICls in combination with targeted therapies, and $43(9.2 \%)$ received dual ICI therapy. Overall, 186 patients (39.5\%) experienced an irAE at any time point. Common irAEs included hypothyroidism $(n=42,22.6 \%)$, rush and pruritus $(n=36,19.4 \%)$, diarrhea/ colitis $(n=35,18.8 \%)$, transaminitis $(n=32,17.2 \%)$, and pneumonitis $(n=14,7.5 \%)$. Monthly incidence rates decreased over time; however, 17 of 109 (15.6\%, 95\% Cl: $9.4 \%$ to $23.8 \%$ ) experienced their first irAE at least 1 year after treatment initiation. No differences in cumulative incidence were observed based on cancer type, agent, or irAE grade. On multivariable analysis, combined ICI therapy with another ICl or with targeted therapy $(p<0.001)$, firstline ICI therapy $(p=0.011)$, and PD- 1 inhibitor therapy $(p=0.007)$ were all significantly associated with irAE development.

Conclusions This study quantitates the incidence of developing irAEs due to ICI conditioned on time elapsed without irAE development. Although the monthly incidence of irAEs decreased over time on therapy, patients can still develop delayed irAEs beyond ICI discontinuation, and thus, continuous vigilant monitoring is warranted.

\section{INTRODUCTION}

Treatment with immune checkpoint inhibitors (ICIs) has transformed the field of oncology, improving long-term survival in patients across several types of cancer. ${ }^{12}$ The most commonly used ICIs target cytotoxic T-lymphocyte-associated antigen 4 (CTLA4 ), programmed cell death protein 1 (PD-1), and PD-ligand 1 (PD-L1). ${ }^{3-5}$ However, these agents are associated with a distinct spectrum of side effects resulting from activation of the immune system, termed immune-related adverse events (irAEs) ${ }^{6-8}$

irAEs can potentially affect any organ system, but most commonly involve the skin, gastrointestinal tract, lungs, liver, and endocrine glands. ${ }^{7-9}$ The incidence of irAEs has been well characterized in the literature with the median time to onset of 2-3 months after initiation. ${ }^{10}{ }^{11}$ Nonetheless, delayed or latent irAEs have been reported months or even years after initiating therapy with onset extending beyond treatment discontinuation. ${ }^{12}$ In clinical trials, the analysis of ICI safety in terms of irAEs is generally reported as incidence proportions. Incidence proportion is typically calculated by crude rates, that is, the ratio of the number of patients who developed the specific adverse event at any point in time to the total number of patients in the cohort. A simple descriptive listing of irAEs in clinical trials is inadequate for modern immunotherapy treatments because this method does not account for the toxicity profile of ICIs conditioned over time. To date, there are limited studies that investigate and quantify the risk of irAEs over time in patients with cancer treated with ICIs. ${ }^{10} 13$ Accurate estimates of this risk will guide oncologists and patients to make decisions regarding treatment strategy and monitoring. 
Here, we performed a retrospective study that evaluated, for the first time, the cumulative incidence of irAEs in patients with metastatic urothelial carcinoma (mUC) and metastatic renal cell carcinoma (mRCC) treated with ICIs. Additionally, we investigated the concept of conditional toxicity, how the incidence of irAE occurrence may be dynamic and may change over time, as well as the risk factors associated with the development of irAEs.

\section{METHODS}

\section{Data Collection}

We conducted a retrospective medical record review of patients with mUC and mRCC who received ICI-based therapy at the Dana Farber Cancer Institute (DFCI) between July 2013 and October 2018. All patients had a histologically confirmed UC or RCC. Patients treated with a CTLA-4 inhibitor and/or PD-1/PD-L1 inhibitor were eligible. Data regarding clinicopathological features and treatment history were extracted. irAEs were defined as adverse events with a potential immunological basis that medical oncologists could recognize objectively. The patients were divided into two groups based on the presence or absence of irAEs within 1 month of each dose of ICI.

Toxicities were graded using the Common Terminology Criteria for Adverse Events (CTCAE) V.5.0. We noted the irAEs and grade reported by the medical professional who encountered a patient experiencing acute irAE. If the grade was not reported, we assigned it based on a thorough review of the medical record describing the events.

\section{Statistical analysis}

Descriptive statistics were used to summarize patient characteristics and outcomes. Time to irAE was defined as the amount of time elapsed from the date of ICI initiation to the development of irAE. The monthly incidence of irAEs was calculated for each 30-day period and was defined as the ratio of the number of patients with an irAE to the number of patients alive without irAE at the start of each month. Similarly, the incidence of irAEs at landmark times was defined as the ratio of the number of patients with an irAE at any time following the landmark time to the number of patients alive at each landmark date who had not experienced a prior irAE. Cumulative incidence was calculated to evaluate the time to the first occurrence of an irAE accounting for the competing risk of death. We used the Fine and Gray method to identify variables associated with irAE development: age at the start of ICI, sex (female vs male), cancer type (mUC vs mRCC), ECOG PS ( 0 vs $\geq 1)$, lung metastases (yes vs no), lymph node metastases (yes vs no), bone metastases (yes vs no), liver metastases (yes vs no), brain metastases (yes vs no), other metastases (yes vs no), ICI type (ICI monotherapy vs dual ICI regimens vs ICI plus another class of agents), line of ICI (first line vs second or more), and type of ICI (PD-L1 inhibitors vs PD-1 inhibitors). A multivariable model was constructed using forward stepwise selection. All tests were two-sided and a p-value of $<0.05$ was considered statistically significant. Statistical analyzes were performed using SAS V.9.2 or R V.3.50 (www.r-project.org).

\section{RESULTS \\ Patient characteristics}

A total of 470 patients received ICIs between July 2013 and October 2018: $271 \mathrm{mRCC}(57.7 \%)$ and $199 \mathrm{mUC}$ $(42.3 \%)$. Baseline patient characteristics are reported in table 1. The median follow-up was 11.9 months (maximum $=8.8$ years). The cohort comprised 342 (72.8\%) men and $128(27.2 \%)$ women, with a median age of 65 years (range: 22-91). Overall, $341(72.5 \%)$ patients received ICI monotherapy, 86 (18.3\%) patients received ICI plus another class of agents (including investigational immunotherapy), and 43 (9.2\%) patients received a dual ICI regimen. Most $(n=312,660.2 \%)$ were treated with anti-PD1-based therapy, whereas a third $(\mathrm{n}=159,330.8 \%)$ received an anti-PD-L1- based regimen. The median duration of therapy was 4.0 (95\% CI: 3.6 to 5.0) months. Two hundred and seven (44\%) patients received ICI as first-line therapy and $263(56 \%)$ received as second or later line therapy. Three hundred and fifty patients $(74.5 \%)$ discontinued ICI due to radiological progression $(\mathrm{n}=226,64.6 \%)$, clinical and radiological progression $(n=43,12.3 \%)$, toxicity $(n=55,15.7 \%)$, clinician preference $(n=19,5.4 \%)$, patient preference $(n=3$, $0.9 \%)$, or unknown reasons $(n=4,1.1 \%)$.

\section{Characteristics and frequency of irAEs}

Any grade irAEs occurred in $186(39.5 \%)$ patients (table 2). Most developed the irAE while on treatment $(n=163,87.6 \%)$, whereas $16(8.6 \%)$ developed the irAE within 1 month of therapy discontinuation, and 7 (3.8\%) developed the irAE $>1$ month after the discontinuation. Only four patients experienced an irAE $>35$ days after therapy discontinuation at $1.7,3.8,5.3$, and 15.4 months, respectively. The most frequently observed irAEs of any grade included hypothyroidism $(\mathrm{n}=42,22.6 \%)$, dermatological toxicity $(n=36,19.4 \%)$, diarrhea/colitis $(n=35$, $18.8 \%)$, transaminitis $(\mathrm{n}=32,17.2 \%)$, and pneumonitis $(\mathrm{n}=14,7.5 \%)$. Fewer than $10 \%(\mathrm{n}=40,8.5 \%)$ exhibited grade 3 irAEs and two patients $(0.4 \%)$ experienced grade 4 events. No treatment-related deaths were reported. The median time to irAE was 21 weeks (range: 0.3-356).

\section{Cumulative incidence of irAEs}

Forty-seven $(10.0 \%)$ patients experienced an irAE within the first month (table 3 ) of receiving ICIs. The monthly incidence of irAEs was lower in subsequent months with $4.2 \%, 5.7 \%$, and $1.4 \%$ developing their first irAE in month 4,7 , and 10 from ICI initiation, respectively. When examining irAEs after landmark times, 139 of $415(33.5 \%)$ patients with no irAE within the first 30 days eventually experienced an irAE. The rates of irAE development after months 3, 6, 9, and 12 were $27.6 \%$ 
Table 1 Baseline patient characteristics $(\mathrm{N}=470)$

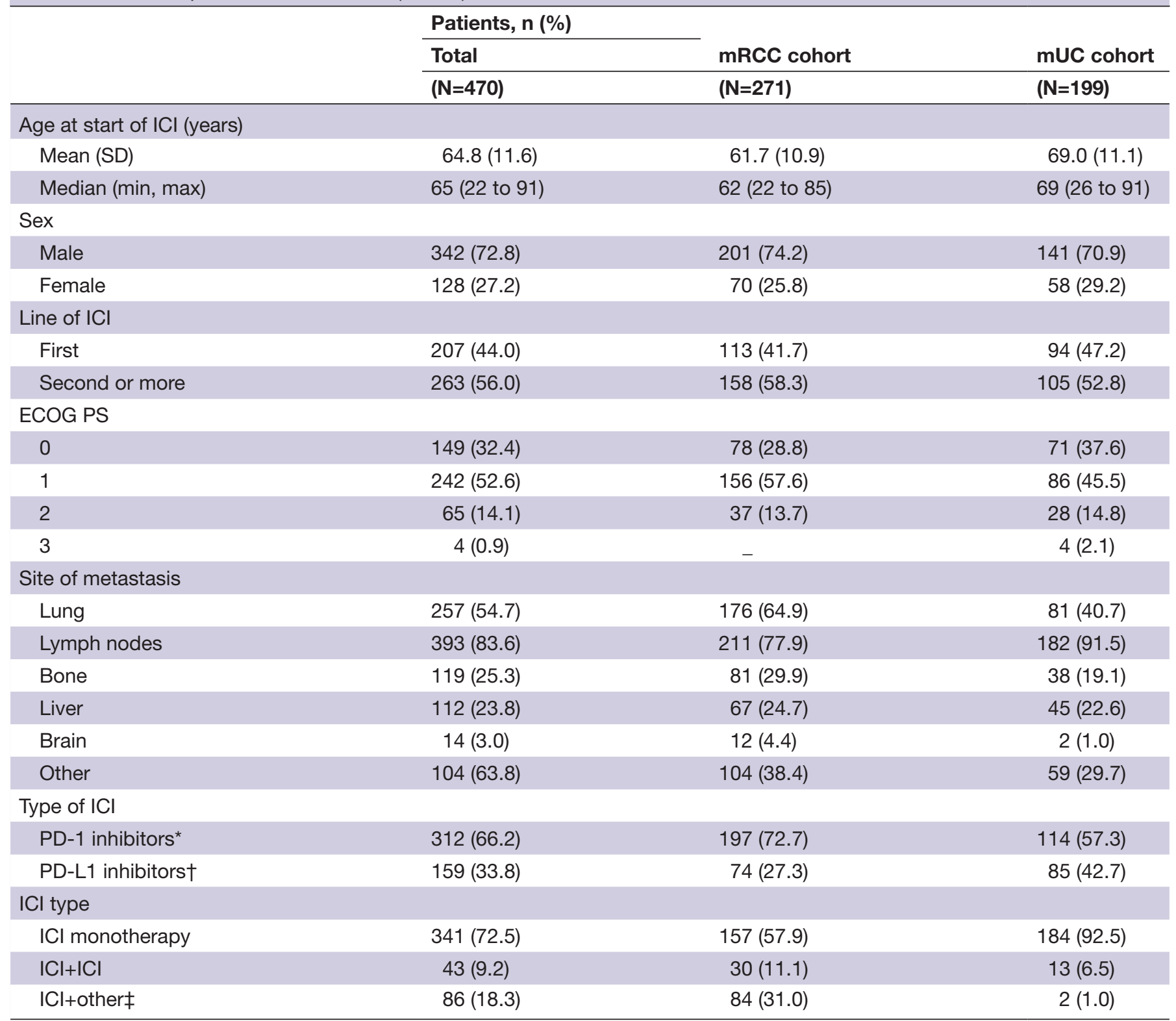

*Includes pembrolizumab and nivolumab.

†Includes atezolizumab, avelumab, and durvalumab.

$\ddagger$ Investigational immunotherapy and targeted therapy agents.

$\mathrm{ICI}$, immune checkpoint inhibitor; mRCC, metastatic renal cell carcinoma; mUC, metastatic urothelial carcinoma; PD-1, programmed cell death protein 1; PD-L1, programmed cell death ligand 1; ECOG PS, Eastern Cooperative Oncology Group Performance Status.

$(\mathrm{n}=86 / 312), 22.3 \%(\mathrm{n}=43 / 193), 14.4 \%(\mathrm{n}=21 / 146)$, and $15.6 \%(\mathrm{n}=17 / 109)$, respectively (table 3$)$. The cumulative incidence of irAE was $32.1 \%(95 \%$ CI: $27.7 \%$ to $36.5 \%)$ at 6 months, $38.8 \%$ (95\% CI: $34.1 \%$ to $43.4 \%)$ at 1 year, and $46.3 \%$ (95\% CI: $40.4 \%$ to $52.2 \%$ ) at 5 years (figure $1 \mathrm{~A}$ ). The cumulative incidence of a grade $\geq 3 \mathrm{irAE}$ was $7.5 \%$ (95\% CI: $5.0 \%$ to $10.0 \%)$ at 6 months, $10.6 \%$ (95\% CI: $7.6 \%$ to $13.6 \%$ ) at 1 year, and $11.7 \%$ (95\% CI: $8.3 \%$ to $15.0 \%$ ) at 5 years (online supplementary table 1 and figure 1B). Cumulative incidence of irAEs for mRCC and mUC were similar for all irAEs, irrespective of grade of toxicity (figure 1).

\section{Characteristics of irAEs conditioned as a function of time}

The type and severity of irAEs experienced after 12 months from ICI initiation were similar to irAEs experienced at any time point. Seventeen of 109 patients (15.6\%) were alive at 12 months after initiating an ICI without irAE and later experienced an irAE beyond 12 months. The most common irAE experienced after 1 year was colitis $(n=5)$, followed by hypothyroidism, rash, pneumonitis, myalgia $(\mathrm{n}=2$ each), joint pain, transaminitis, uveitis, and nephritis $(n=1$ each $)$. In terms of severity, most were grade $1-2$ with one grade 4 colitis and one grade 3 myalgia. 
Open access

Table 2 irAEs according to category and grade

\begin{tabular}{|c|c|c|c|c|}
\hline \multirow[b]{2}{*}{ Category } & \multirow{2}{*}{$\begin{array}{l}\text { Patients, n (\%) } \\
\text { Total }\end{array}$} & \multirow[b]{2}{*}{ Grade 1-2 } & \multirow[b]{2}{*}{ Grade 3-4 } & \multirow{2}{*}{$\begin{array}{l}\text { Weeks to onset, } \\
\text { median (range) }\end{array}$} \\
\hline & & & & \\
\hline Any & $186(39.5)$ & $144(30.6)$ & $42(8.9)$ & $21(0.3-356)$ \\
\hline Rash+pruritus & $36(7.7)$ & $31(6.6)$ & $5(1.1)$ & $7(2-100)$ \\
\hline Mucositis & $2(0.4)$ & $1(0.2)$ & $1(0.2)$ & $23(21-25)$ \\
\hline \multicolumn{5}{|l|}{ Endocrine } \\
\hline Hypothyroid & $42(8.9)$ & $42(8.9)$ & 0 & $13(1-154)$ \\
\hline Adrenal insufficiency & $1(0.2)$ & $1(0.2)$ & 0 & 10 \\
\hline \multicolumn{5}{|l|}{ Gastroentelorogical } \\
\hline \multicolumn{5}{|l|}{ Hepatobiliary } \\
\hline \multicolumn{5}{|c|}{ Rheumatological/musculoskeletal } \\
\hline Joint pain & $12(2.6)$ & $11(2.3)$ & $1(0.2)$ & $9(1-67)$ \\
\hline Myalgia & $5(1.1)$ & $4(0.9)$ & $1(0.2)$ & $25(2-64)$ \\
\hline \multicolumn{5}{|l|}{ Others } \\
\hline Nephritis & $3(0.6)$ & $2(0.4)$ & $1(0.2)$ & $17(1-64)$ \\
\hline Pancreatitis & $2(0.4)$ & 0 & $2(0.4)$ & $21(16-25)$ \\
\hline Pericarditis & $1(0.2)$ & $1(0.2)$ & 0 & 8 \\
\hline Uveitis & $1(0.2)$ & $1(0.2)$ & 0 & 56 \\
\hline
\end{tabular}

irAEs, immune-related adverse events.

Risk factors associated with irAE development

The risk factors associated with the development of irAEs at any time point are shown in the online supplementary table 2. In multivariable analysis, combined ICI therapy $(\mathrm{HR}=1.83,95 \% \mathrm{CI}: 1.15$ to 2.91$)$ and combined ICI plus other agents $(\mathrm{HR}=2.29,95 \% \mathrm{CI}: 1.55$ to 3.39$)$ versus IO monotherapy $(\mathrm{p}<0.001)$, first-line versus later-line therapy $(\mathrm{HR}=1.29,95 \% \mathrm{CI}: 1.06$ to $1.56, \mathrm{p}=0.011)$, and PD-1 versus PD-L1 inhibitor therapy $(\mathrm{HR}=1.64,95 \% \mathrm{CI}$ : 1.15 to $2.36, \mathrm{p}=0.007$ ) were all significantly associated

Table 3 Monthly incidence and conditional probability of irAEs

\begin{tabular}{lllll}
\hline Landmark month & $\begin{array}{l}\text { Patients alive on landmark day } \\
\text { with no prior irAE, } \mathbf{N}\end{array}$ & $\begin{array}{l}\text { Patients with irAEs in } \\
\text { next } \mathbf{3 0} \text { days, } \mathbf{n} \text { (\%) }\end{array}$ & $\begin{array}{l}\text { Patients with irAEs at any } \begin{array}{l}\text { 95\% } \mathbf{C l} \text { for future } \\
\text { time in the future, } \mathbf{n}(\%)\end{array} \\
\text { irAE at any time }\end{array}$ \\
\hline 0 & 470 & $47(10.0)$ & $186(39.6)$ & 35.1 to 44.2 \\
\hline 1 & 415 & $28(6.8)$ & $139(33.5)$ & 29.0 to 38.3 \\
\hline 2 & 358 & $25(7.0)$ & $111(31.0)$ & 26.3 to 36.1 \\
\hline 3 & 312 & $13(4.2)$ & $86(27.6)$ & 22.7 to 32.9 \\
\hline 4 & 270 & $19(7.0)$ & $73(27.0)$ & 18.8 to 30.5 \\
\hline 5 & 222 & $11(5.0)$ & $54(24.3)$ & 16.6 to 28.8 \\
\hline 6 & 193 & $11(5.7)$ & $43(22.3)$ & 12.8 to 24.7 \\
\hline 7 & 176 & $5(2.8)$ & $32(18.2)$ & 11.2 to 23.2 \\
\hline 8 & 163 & $6(3.7)$ & $27(16.6)$ & 9.1 to 21.1 \\
\hline 10 & 146 & $2(1.4)$ & $21(14.4)$ & 8.9 to 21.6 \\
\hline 11 & 132 & $2(1.5)$ & $19(14.4)$ & 8.6 to 22.1 \\
\hline
\end{tabular}

$\mathrm{Cl}$, cumulative incidence; irAEs, immune-related adverse events. 


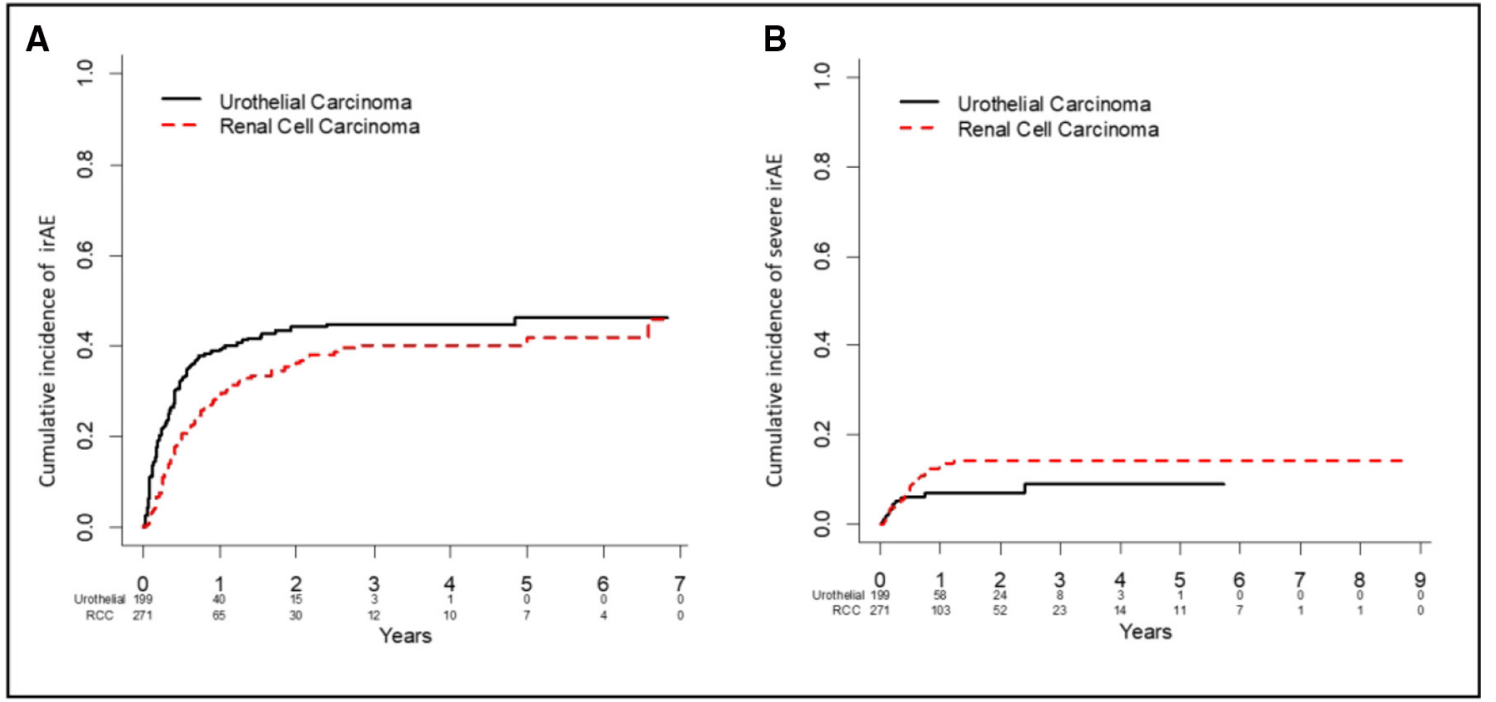

Figure 1 Cumulative incidence irAEs plots for $m R C C$ and $m U C$ : irAEs all grades (A), irAEs grades $\geq 3$ (B). The cumulative incidence of irAEs for $\mathrm{mRCC}$ and $\mathrm{mUC}$ were similar for all irAEs, irrespective of grade of toxicity. The cumulative incidence of irAE was $32.1 \%(95 \% \mathrm{Cl}$ : $27.7 \%$ to $36.5 \%)$ at 6 months, $38.8 \%(95 \% \mathrm{Cl}: 34.1 \%$ to $43.4 \%)$ at 1 year, and $46.3 \%$ (95\% $\mathrm{Cl}: 40.4 \%$ to $52.2 \%)$ at 5 years (A). The cumulative incidence of a grade $\geq 3$ irAE was $7.5 \%(95 \% \mathrm{Cl}: 5.0 \%$ to $10.0 \%)$ at 6 months, $10.6 \%$ (95\% Cl: $7.6 \%$ to $13.6 \%)$ at 1 year, and $11.7 \%(95 \% \mathrm{Cl}: 8.3 \%$ to $15.0 \%)$ at 5 years (B). irAE, immune-related adverseevent; $\mathrm{mRCC}$, metastatic urothelialcarcinoma; mUC, metastatic renal cell carcinoma.

with irAE development. Type of cancer, age, sex, and performance status were not associated with a higher risk of irAE development.

\section{DISCUSSION}

Conventional safety analyzes describing the absolute percentages of adverse events experienced by patients do not account for how the toxicity profile and risk may evolve over time. In this study, we retrospectively analyzed 470 patients including 271 patients with mRCC and 199 patients with mUC who had received ICI-based therapy at a single academic institution with the objective of characterizing the cumulative incidence of irAEs and assessing how risk may change when conditioned on time elapsed. Our results suggest that the monthly risk of developing an irAE persists but decreases over time, irrespective of the type of cancer, specific ICI class, and known prognostic clinical or demographic variables. Our study highlights that when examining the monthly incidence of irAEs, patients are at the highest risk of developing the irAE in the first month of treatment (10\%). Although still at risk for developing irAEs during the remaining months of the first year, there was a trend for decreased monthly incidence with time elapsed. Notably, a substantial proportion $(15.6 \%)$ of patients experienced delayed irAEs after 1 year of treatment. To our knowledge, this is the first study to underscore the important role of time-to-adverse event analysis in the setting of ICIs, which could inform clinical practice and trial designs.

Few studies have investigated the incidence of irAEs with ICI and how the risk may change over time during treatment. ${ }^{1012} 13$ In a safety subanalysis of one prospective study, prolonged treatment with ICIs did not result in an increased cumulative incidence of irAEs. ${ }^{10}$ The safety report of 107 patients with advanced melanoma treated with nivolumab showed that most irAEs occurred within the first 6 months, and cumulative toxicities were not observed with prolonged drug exposure, although details about the type, grade, and incidence of irAE over time were not reported. ${ }^{10}$ In a retrospective review including 352 patients enrolled in 21 early phase clinical trials of ICI, $260(74 \%)$ patients experienced irAEs. ${ }^{13}$ In this case, the risk of developing irAEs was studied using the OR model for irAEs occurrence at various time points. ${ }^{13}$ The OR for irAEs occurrence within the first month compared with after 1 month was 3.13 (95\% CI: 1.95 to 5.02 ) and decreased over time, being 2.80 (95\% CI: 1.76 to 4.44$)$ at 3 months. ${ }^{13}$

Clinical trials typically include a summary table of adverse events incidence irrespective of when the patient experienced the event. This method of reporting toxicities is suboptimal for capturing the impact of time on therapy free from toxicities, especially when that toxicity can be an important cause of treatment discontinuation in patients receiving ICIs. Quantification of irAE risk over time offers important information for clinical decision making for the patient's treatment strategy. PD-1/-L1 inhibitors are administered continuously over months to years in patients with the stable or responding disease, rather than given for a predefined number of cycles. In contrast to cytotoxic chemotherapy, where the adverse events typically occur early and accumulate during the treatment course, toxicity with ICI may manifest later and thus, clinicians and patients should continue to be vigilant especially in situations of prolonged therapy. Incorporating the cumulative incidence of irAEs in clinical 
trials is an opportunity to improve the characterization of toxicities and tailoring of monitoring schedule as well as in counseling of patients in the clinic.

It is recognized that irAEs exhibit a characteristic pattern in timing in their occurrence, with some events, like skin, hepatic, and gastrointestinal toxicities, occurring earlier at approximately $2-6$ weeks, and pneumonitis or endocrine events occurring on average 9 weeks after treatment initiation. ${ }^{11}$ In our dataset, when we examined irAEs that occurred within 1 year vs $>1$ year, no differences in the irAE class emerged, but our analysis may be underpowered. The precise pathophysiology that explains the relatively high rate of late irAEs occurring after 1 year remains unclear. One hypothesis is that early irAEs occur by the cross-reactivity theory, where the unbalancing of the immune system induced by ICI generates a crossreactivity between tumor neoantigens and normal tissue antigens. ${ }^{14} 15$ Another theory postulates that ICIs can modulate B cells over time both directly and indirectly via T-cell mediation, altering the production of autoantibodies that can generate delayed irAEs. ${ }^{16}{ }^{17}$ Non-specific increase in endogenous T-cell response mediated by dendritic cell or cytokine stimulation could play a role in this process. ${ }^{16}$ Furthermore, ICIs have a serum half-life of 12-20 days and therefore they can continue to inhibit immune checkpoint pathways for $>8$ months after the last dose. $^{18}$

The fact that the cumulative incidence rates of irAEs were similar in mRCC and $\mathrm{mUC}$ should not be surprizing as the pathophysiology of irAEs is anticipated to be driven by host genetics and not tumor biology. ${ }^{19}$ Interestingly, the rate of grade $\geq 3$ irAEs was only $8.5 \%$ despite an overall irAE rate of $39.5 \%$, which suggests that prompt recognition and management of irAEs possible in an academic tertiary care center, may mitigate the risk of severe irAEs. Moreover, we showed that the type of ICI or drug combination did not affect the cumulative incidence, suggesting that the trend for safety over time is independent of the type of ICI. As expected, ICI combination therapy was associated with a higher incidence of irAEs. ${ }^{20-23}$ PD-1 inhibitors have been previously reported to carry a higher risk of toxicities compared with PD-L1 inhibitors, and our findings are consistent with these data. ${ }^{24}$ The association of first-line therapy with a higher risk of irAEs compared with later line settings is intriguing and has not been reported before to our knowledge. This result is biologically plausible given a likely more intact host immune system leading to a more robust systemic immune response in treatment-naïve or less advanced disease patients. However, these results require validation and conclusions are limited pending further understanding of host genetic factors or other host or tumor biologic contributors that may increase the risk of irAEs.

Limitations of our study include its retrospective nature. Patients were all treated at a single institution, so it is possible that results may not be completely generalizable to other institutions with different practice patterns or patient populations. Second, we relied on the clinical information reported in the charts for the identification and grading, of irAEs and thus some irAEs may have been misclassified or not reported. However, this risk is lessened by the execution of the study at a major academic institution with investigators who are highly experienced in the recognition and management of irAEs. Third, the frequency and type of irAEs may vary depending on the specific ICI treatment; thus, a different irAE profile may be observed with different ICI regimens. Differences in the cumulative incidence rate may be seen based on specific agents or combinations, but we did not have the power to examine these subsets separately beyond combination versus monotherapy analyzes. We believe the rates of irAEs, in particular serious irAEs, while slightly lower than many trials, are similar and as expected. Moreover, given the greater awareness of irAEs following the publication of multiple trials in different malignancies, toxicities may have been more promptly managed leading to a somewhat lower rate of severe irAEs. We examined only data from metastatic RCC and UC, which was available to us; however, the consistent results of our analysis regardless of malignancy suggests that the type of malignancy does not impact conditional irAE rates.

\section{CONCLUSIONS}

In conclusion, this is the first retrospective study that quantitates the incidence of developing irAEs with ICI conditioned on time elapsed in a cohort of patients with mRCC and mUC treated with ICIs. Our results highlight that the monthly incidence of irAEs decreases over time on therapy; however, a significant proportion of delayed irAEs occur even $\geq 1$ year after initiating ICIs. Thus, awareness and ongoing vigilant monitoring is required throughout ICI therapy and likely beyond discontinuation. In conjunction with an investigation into clinical and biologic predictors of early and late irAEs, incorporation of conditional irAE rates is warranted in ongoing and future immunotherapy studies to enhance our understanding of ICI toxicity profile and optimize patient care.

\section{Author affiliations}

${ }^{1}$ Department of Medical Oncology, Dana Farber Cancer Institute, Boston,

Massachusetts, USA

${ }^{2}$ Department of Oncology, McMaster University, Hamilton, Ontario, Canada ${ }^{3}$ Department of Medicine, Brigham and Women's Hospital, Boston, Massachusetts, USA

Twitter Pier Vitale Nuzzo @PierVitaleNuzzo, Amin H Nassar @AminNassarMD, Toni Choueiri @DrChoueiri and Guru Sonpavde @sonpavde

Acknowledgements The auhtors thank the patients for their contributions to research.

Contributors PVN, GRP and GS: conception and design. PVN, GRP, SAA, AHN, RF, KK, XXW, BAM, LH, TKC, and GS:development and methodology. PVN, SAA, AHN, RF, KK, XXW, BAM, LH, TKC and CC: collection clinical data. GSP: statistical analysis. PVN, GRP, SAA, AHN, RF, KK, XXW, BAM, LH, TKC and GS: analysis and interpretation of data. PVN and CC: administrative, technical, or material support. GS: study supervision. All authors: writing, review and/or revision of the manuscripts; read and approved the final manuscript.

Funding The authors have not declared a specific grant for this research from any funding agency in the public, commercial or not-for-profit sectors. 
Competing interests None declared.

Patient consent for publication Written informed consent was obtained from patients to collect clinical information.

Ethics approval This study was approved by the Institutional Review Board at Dana Farber Cancer Institute.

Provenance and peer review Not commissioned; externally peer reviewed.

Data availability statement Data are available upon reasonable request. All data relevant to the study are included in the article or uploaded as supplementary information. All data and materials relevant to this article are available to referees at submission and to readers promptly upon request

Open access This is an open access article distributed in accordance with the Creative Commons Attribution Non Commercial (CC BY-NC 4.0) license, which permits others to distribute, remix, adapt, build upon this work non-commercially, and license their derivative works on different terms, provided the original work is properly cited, appropriate credit is given, any changes made indicated, and the use is non-commercial. See http://creativecommons.org/licenses/by-nc/4.0/.

\section{ORCID iDs}

Pier Vitale Nuzzo http://orcid.org/0000-0002-5618-8079

Amin H Nassar http://orcid.org/0000-0002-8084-9105

Toni Choueiri http://orcid.org/0000-0002-9201-3217

Lauren C Harshman http://orcid.org/0000-0002-7636-1588

\section{REFERENCES}

1 Xu C, Chen Y-P, Du X-J, et al. Comparative safety of immune checkpoint inhibitors in cancer: systematic review and network metaanalysis. BMJ 2018;363:k4226.

2 Wei Y, Du Q, Jiang X, et al. Efficacy and safety of combination immunotherapy for malignant solid tumors: a systematic review and meta-analysis. Crit Rev Oncol Hematol 2019;138:178-89.

3 Dong H, Strome SE, Salomao DR, et al. Tumor-Associated B7H1 promotes T-cell apoptosis: a potential mechanism of immune evasion. Nat Med 2002;8:793-800.

4 Boussiotis VA. Molecular and biochemical aspects of the PD-1 checkpoint pathway. N Engl J Med 2016;375:1767-78.

5 Tivol EA, Borriello F, Schweitzer AN, et al. Loss of CTLA-4 leads to massive lymphoproliferation and fatal multiorgan tissue destruction, revealing a critical negative regulatory role of CTLA-4. Immunity 1995;3:541-7.

6 Postow MA, Sidlow R, Hellmann MD. Immune-Related adverse events associated with immune checkpoint blockade. $N$ Engl J Med 2018;378:158-68.

7 Brahmer JR, Lacchetti C, Schneider BJ, et al. Management of immune-related adverse events in patients treated with immune checkpoint inhibitor therapy: American Society of clinical oncology clinical practice guideline. J Clin Oncol 2018;36:1714-68.
8 Puzanov I, Diab A, Abdallah K, et al. Managing toxicities associated with immune checkpoint inhibitors: consensus recommendations from the Society for immunotherapy of cancer (SITC) toxicity management Working group. J Immunother Cancer 2017;5:95.

9 Stucci S, Palmirotta R, Passarelli A, et al. Immune-Related adverse events during anticancer immunotherapy: pathogenesis and management. Oncol Lett 2017;14:5671-80.

10 Topalian SL, Sznol M, McDermott DF, et al. Survival, durable tumor remission, and long-term safety in patients with advanced melanoma receiving nivolumab. J Clin Oncol 2014;32:1020-30.

11 Weber JS, Dummer R, de Pril V, Pril de V, et al. Patterns of onset and resolution of immune-related adverse events of special interest with ipilimumab: detailed safety analysis from a phase 3 trial in patients with advanced melanoma. Cancer 2013;119:1675-82.

12 Couey MA, Bell RB, Patel AA, et al. Delayed immune-related events (dire) after discontinuation of immunotherapy: diagnostic hazard of autoimmunity at a distance. J Immunother Cancer 2019;7:165.

13 Kanjanapan Y, Day D, Butler MO, et al. Delayed immune-related adverse events in assessment for dose-limiting toxicity in early phase immunotherapy trials. Eur J Cancer 2019;107:1-7.

14 Huang SK, Okamoto T, Morton DL, et al. Antibody responses to melanoma/melanocyte autoantigens in melanoma patients. J Invest Dermatol 1998;111:662-7.

15 Cheng F, Loscalzo J. Autoimmune cardiotoxicity of cancer immunotherapy. Trends Immunol 2017;38:77-8.

16 Ueda H, Howson JMM, Esposito L, et al. Association of the T-cell regulatory gene CTLA4 with susceptibility to autoimmune disease. Nature 2003;423:506-11.

17 Prokunina L, Castillejo-López C, Öberg F, et al. A regulatory polymorphism in PDCD1 is associated with susceptibility to systemic lupus erythematosus in humans. Nat Genet 2002;32:666-9.

18 Brahmer JR, Drake CG, Wollner I, et al. Phase I study of single-agent anti-programmed death-1 (MDX-1106) in refractory solid tumors: safety, clinical activity, pharmacodynamics, and immunologic correlates. J Clin Oncol 2010;28:3167-75.

19 Atamaniuk J, Kopecky C, Skoupy S, et al. Apoptotic cell-free DNA promotes inflammation in haemodialysis patients. Nephrol Dial Transplant 2012;27:902-5.

20 Bertrand A, Kostine M, Barnetche T, et al. Immune related adverse events associated with anti-CTLA-4 antibodies: systematic review and meta-analysis. BMC Med 2015;13:211.

21 Maughan BL, Bailey E, Gill DM, et al. Incidence of immune-related adverse events with program death receptor-1- and program death receptor-1 ligand-directed therapies in genitourinary cancers. Front Oncol 2017;7:56.

22 Wolchok JD, Chiarion-Sileni V, Gonzalez R, et al. Overall survival with combined nivolumab and ipilimumab in advanced melanoma. $N$ Engl J Med 2017;377:1345-56.

23 Zhou S, Khanal S, Zhang H. Risk of immune-related adverse events associated with ipilimumab-plus-nivolumab and nivolumab therapy in cancer patients. Ther Clin Risk Manag 2019;15:211-21.

24 Wang Y, Zhou S, Yang F, et al. Treatment-Related adverse events of PD-1 and PD-L1 inhibitors in clinical trials: a systematic review and meta-analysis. JAMA Oncol 2019;5 